\title{
CAT(0) spaces with boundary the join of two Cantor sets
}

\author{
KHEK LUN HAROLD CHAO
}

\begin{abstract}
We will show that if a proper complete CAT(0) space $X$ has a visual boundary homeomorphic to the join of two Cantor sets, and $X$ admits a geometric group action by a group containing a subgroup isomorphic to $\mathbb{Z}^{2}$, then its Tits boundary is the spherical join of two uncountable discrete sets. If $X$ is geodesically complete, then $X$ is a product, and the group has a finite index subgroup isomorphic to a lattice in the product of two isometry groups of bounded valence bushy trees.
\end{abstract}

20F65; 20F67, 51F99

\section{Introduction}

CAT(0) spaces with homeomorphic visual boundaries can have very different Tits boundaries. However, if $X$ admits a proper and cocompact group action by isometries, or a geometric group action in short, then this places a restriction on the possible Tits boundaries for a given visual boundary. (We follow the definition of a proper group action in Bridson-Haefliger [3, Chapter I.8]; some use the term "properly discontinuous" for this.) Kim Ruane has showed in [13] that for a CAT(0) space $X$ with boundary $\partial X$ homeomorphic to the suspension of a Cantor set, if it admits a geometric group action, then the Tits boundary $\partial_{\mathrm{T}} X$ is isometric to the suspension of an uncountable discrete set. In this paper we will show the following.

Theorem 1.1 If a CAT(0) space $X$ has a boundary $\partial X$ homeomorphic to the join of two Cantor sets $C_{1}$ and $C_{2}$ and if $X$ admits a geometric group action by a group containing a subgroup isomorphic to $\mathbb{Z}^{2}$, then its Tits boundary $\partial_{\mathrm{T}} X$ is isometric to the spherical join of two uncountable discrete sets. So if $X$ is geodesically complete, then $X=X_{1} \times X_{2}$ with $\partial X_{i}$ homeomorphic to $C_{i}, i=1,2$.

As for the group acting on $X$, we will prove the following.

Theorem 1.2 Let $X$ be a geodesically complete CAT(0) space such that $\partial X$ is homeomorphic to the join of two Cantor sets. Then for a group $G<\operatorname{Isom}(X)$ acting 
geometrically on $X$ and containing a subgroup isomorphic to $\mathbb{Z}^{2}$, either $G$ or a subgroup of $G$ of index 2 is a uniform lattice in $\operatorname{Isom}\left(X_{1}\right) \times \operatorname{Isom}\left(X_{2}\right)$. Furthermore, a finite index subgroup of $G$ is a lattice in $\operatorname{Isom}\left(T_{1}\right) \times \operatorname{Isom}\left(T_{2}\right)$, where $T_{i}$ is a bounded valence bushy tree quasi-isometric to $X_{i}, i=1,2$.

Remark The assumption that $G$ contains a subgroup isomorphic to $\mathbb{Z}^{2}$ is only used to obtain a hyperbolic element in $G$ with endpoints in $\partial X \backslash\left(C_{1} \cup C_{2}\right)$, which we use in Section 4 to prove Theorem 1.1. It is conjectured that a CAT $(0)$ group is either Gromov hyperbolic or it contains a subgroup isomorphic to $\mathbb{Z}^{2}$. Without using the assumption on $G$, we can show that $G$ cannot be hyperbolic, which follows from Lemma 2.3 and the flat plane theorem [3, Theorem III.H.1.5]. Thus if the conjecture is shown to be true for general CAT(0) groups, the assumption on $G$ will not be necessary. The conjecture has been proved for some classes of CAT(0) groups; see Kapovich-Klein [8] and Caprace-Haglund [5] for examples.

If $X_{i}$ are proper geodesically complete, one might hope that they are trees, so $G$ will be a uniform lattice in the product of two isometry groups of trees. Surprisingly, this may not be the case. Ontaneda constructed a 2-complex $Z$ which is non-positively curved and geodesically complete with free group $F_{n}$ as its fundamental group (see Ontaneda [10, Proposition 1]). Its universal cover is quasi-isometric to $F_{n}$, so it is a Gromov hyperbolic space with Cantor set boundary, while being also a CAT(0) space. Under an additional condition that the isotropy subgroup of $\operatorname{Isom}\left(X_{i}\right)$ of every boundary point of $X_{i}$ acts cocompactly on $X_{i}$, then $X_{i}$ is a tree (see Caprace-Monod [6, Theorem 1.3]).

There are irreducible lattices in a product of two trees, so $G$ may not have a finite index subgroup which splits as a product. See Burger-Mozes [4] for a detailed investigation.

Acknowledgement I would like to thank my advisor Chris Connell for suggesting this problem to me and providing me with a lot of valuable discussions, assistance and encouragements while I was working on this project.

\section{Preliminaries}

First we fix the notations. For a $\operatorname{CAT}(0)$ space $X$, its (visual) boundary with the cone topology is $\partial X$. For a subset $H \subset X$, we denote by $\partial H:=\bar{H} \cap \partial X$, where the closure $\bar{H}$ is taken in $\bar{X}:=X \cup \partial X$. The angular and the Tits metrics on the boundary are denoted as $\angle(\cdot, \cdot)$ and $\mathrm{d}_{\mathrm{T}}(\cdot, \cdot)$ respectively. We denote the boundary with the Tits metric by $\partial_{\mathrm{T}} X$. The identity map from the Tits boundary $\partial_{\mathrm{T}} X$ to the 
visual boundary $\partial X$ is continuous but usually not a homeomorphism (see BridsonHaefliger [3, Proposition II.9.7]). If $g$ is a group element acting on $X$ by isometry, we denote by $\bar{g}$ the action of $g$ extended to $\partial X$ by homeomorphism. If $g$ acts on $X$ by a hyperbolic isometry, the two endpoints of its axes on $\partial X$ are denoted by $g^{ \pm \infty}$. We refer to [3] for details on basic facts about CAT(0) spaces.

Let $X$ be a complete CAT( 0$)$ space with $\partial X$ homeomorphic to the join of two Cantor sets $C_{1}$ and $C_{2}$, and $G<\operatorname{Isom}(X)$ be a group acting on $X$ geometrically. We will not assume that $G$ contains a subgroup isomorphic to $\mathbb{Z}^{2}$ until Section 4 . By the following lemma, we can assume that $G$ stabilizes $C_{1}$ and $C_{2}$.

Lemma 2.1 Either $G$ or a subgroup of $G$ of index 2 stabilizes each of $C_{1}$ and $C_{2}$.

Proof Consider $\partial X$ as a complete bipartite graph with $C_{1}, C_{2}$ as the two sets of vertices. For any $g \in G$, if $\bar{g} \cdot x_{1} \in C_{1}$ for some $x_{1} \in C_{1}$, then $\bar{g} \cdot C_{i}=C_{i}, i=1,2$; otherwise $\bar{g} \cdot C_{1}=C_{2}$ and $\bar{g} \cdot C_{2}=C_{1}$. So the homomorphism from $G$ to symmetric group on two elements is well-defined and its kernel is the subgroup of $G$ which stabilizes each of $C_{1}$ and $C_{2}$.

By an arc we specifically mean a segment from a point in $C_{1}$ to a point in $C_{2}$ which does not pass through any other point of $C_{1}$ or $C_{2}$, and by open (closed) segment a segment on the boundary excluding (including) its two endpoints. We will investigate the positions of the endpoints of hyperbolic elements in $G$.

We quote a basic result on dynamics on $\operatorname{CAT}(0)$ space boundary by Ruane:

Lemma 2.2 (Ruane [12, Lemma 4.1]) Let $g$ be a hyperbolic isometry of a CAT(0) space $X$ and let $c$ be an axis of $g$. Let $z \in \partial X, z \neq g^{-\infty}$ and let $z_{i}=\bar{g}^{i} \cdot z$. If $w \in \partial X$ is an accumulation point of the sequence $\left(z_{i}\right)$ in the cone topology, then $\angle\left(g^{-\infty}, w\right)+\angle\left(w, g^{\infty}\right)=\pi$, and $\angle\left(g^{-\infty}, z\right)=\angle\left(g^{-\infty}, w\right)$. If $w \neq g^{\infty}$, then $\mathrm{d}_{\mathrm{T}}\left(g^{-\infty}, w\right)+\mathrm{d}_{\mathrm{T}}\left(w, g^{\infty}\right)=\pi$. In this case $c$ and a ray from $c(0)$ to $w$ span a flat half plane, and $\mathrm{d}_{\mathrm{T}}\left(g^{-\infty}, z\right)=\mathrm{d}_{\mathrm{T}}\left(g^{-\infty}, w\right)$.

Recall that a hyperbolic isometry is of rank one if none of its axes bounds a flat half plane, and it is of higher rank otherwise.

Lemma 2.3 There is no rank-one isometry in $G$.

Proof Take any hyperbolic $g \in G$. Assume without loss of generality that $g^{\infty} \in$ $\partial X \backslash C_{2}$. Then for any point $y \in C_{2}, \bar{g}^{n} \cdot y$ cannot accumulate at $g^{\infty}$ since $C_{2}$ is closed in $\partial X$. Any accumulation point of $\bar{g}^{n} \cdot y$ will form a boundary of a half plane with $g^{ \pm \infty}$ by Lemma 2.2. So $g$ is not rank one. 
We note also that no finite subset of points on the boundary is stabilized by $G$, which readily follows from a result by Ruane, quoted in a paper by Papasoglu and Swenson, and the fact that our $\partial X$ is not a suspension.

Lemma 2.4 (Ruane, Papasoglu-Swenson [11, Lemma 26]) If $G$ virtually stabilizes a finite subset $A$ of $\partial X$, then $G$ virtually has $\mathbb{Z}$ as a direct factor. In this case $\partial X$ is a suspension.

\section{Endpoints of a hyperbolic element}

We will show that there is no hyperbolic element of $G$ with one of its endpoints in $C_{1}$ but not the other one. We will proceed by contradiction, using as a key result the following theorem by Papasoglu and Swenson to $\partial X$, itself a strengthening of a previous result by Ballmann and Buyalo [2]. This theorem is applicable to our $\partial X$ in light of the previous lemmas.

Theorem 3.1 (Papasoglu and Swenson [11, Theorem 22]) If the Tits diameter of $\partial X$ is bigger than $\frac{3 \pi}{2}$ then $G$ contains a rank 1 hyperbolic element. In particular: If $G$ does not fix a point of $\partial X$ and does not have rank 1, and $I$ is a (minimal) closed invariant set for the action of $G$ on $\partial X$, then for any $x \in \partial X, \mathrm{~d}_{\mathrm{T}}(x, I) \leq \frac{\pi}{2}$.

We put the word minimal in parentheses as it is not a necessary condition, for if $I \subset \partial X$ is a closed invariant set, then it contains a minimal closed invariant set $I^{\prime}$, and so for any $x \in \partial X, \mathrm{~d}_{\mathrm{T}}(x, I) \leq \mathrm{d}_{\mathrm{T}}\left(x, I^{\prime}\right) \leq \frac{\pi}{2}$.

Note that the above theorem implies that $\partial X$ has finite Tits diameter, and hence the CAT(1) space $\partial_{\mathrm{T}} X$ is connected.

Now assume that $g \in G$ is hyperbolic such that $g^{\infty} \in C_{1}$ and $g^{-\infty} \in \partial X \backslash C_{1}$.

Lemma 3.2 Fix $(\bar{g})$ contains boundary of a $2-f l a t$.

Proof Since $\bar{g}$ acts on $\partial_{\mathrm{T}} X$ by isometry and $\partial_{\mathrm{T}} X$ is connected, if $g^{-\infty} \in C_{2}$, then the arc between $g^{\infty}$ and $g^{-\infty}$ is fixed by $\bar{g}$; otherwise $g^{-\infty} \notin C_{1} \cup C_{2}$, then $g^{-\infty}$ lies on an open arc joining a point in $C_{1}$ to a point in $C_{2}$, so this arc is fixed by $\bar{g}$. Hence in both cases there is an arc contained in $\partial \operatorname{Min}(g)$. Then by [12, Theorems 3.2 and 3.3], $\operatorname{Min}(g)=Y \times \mathbb{R}, \partial \operatorname{Min}(g)=\operatorname{Fix}(\bar{g})$ and is the suspension of $\partial Y$, and $Z_{g} /\langle g\rangle$ acts on the CAT(0) space $Y$ geometrically. Here we have $\partial Y \neq \varnothing$, for otherwise $\partial \operatorname{Min}(g)$ would consist of only two points. Since $Y$ has nonempty boundary, so by Swenson [14, Theorem 11] there is a hyperbolic element in $Z_{g} /\langle g\rangle$ which has an axis in $Y$ with two endpoints on $\partial Y$. Thus there is a 2-flat in $\operatorname{Min}(g)$. 


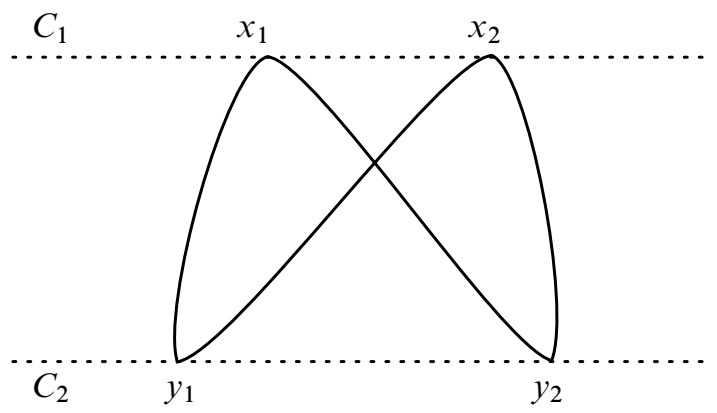

Figure 1: Boundary of a 2-flat in $\operatorname{Min}(h)$

Denote this 2-flat by $F$, and let $z$ be a point in $\partial F \cap C_{1}$ other than $g^{\infty}$.

Lemma 3.3 If $F_{0}$ is a 2-flat whose boundary is contained in $\operatorname{Fix}(\bar{h})=\partial \operatorname{Min}(h)$ for some hyperbolic $h \in G$, then $\partial F_{0}$ intersects each of $C_{1}$ and $C_{2}$ at exactly 2 points.

Proof Suppose not, then denote the points at which $\partial F_{0}$ alternatively intersects $C_{1}$, $C_{2}$ by $x_{1}, y_{1}, x_{2}, y_{2}, \ldots, x_{n}, y_{n}$. Consider the segment joining $x_{1}$ and $y_{2}$. We may assume that not both of $x_{1}, y_{2}$ are endpoints of $h$. (If not, choose $y_{1}$ and $x_{3}$ instead.) From the assumption on $\partial F_{0}$, this segment is not part of $\partial F_{0}$. Its two endpoints are fixed, but the arc joining them is not in $\operatorname{Fix}(\bar{h})$ because $\operatorname{Fix}(\bar{h})$ is a suspension with suspension points $h^{ \pm \infty}$. However, this arc is stabilized by $\bar{h}$ because of the cone topology of $\partial X$. Take a point $p$ in the open arc between $x_{1}$ and $y_{2}$. Since $\partial_{\mathrm{T}} X$ is connected there exists a Tits segment in this arc from $p$ to one of $x_{1}$ and $y_{2}$, say $x_{1}$. The action of $G$ on $\partial_{\mathrm{T}} X$ is by isometries. Choose a new point on this segment as $p$ if necessary, we can assume $\mathrm{d}_{\mathrm{T}}\left(p, x_{1}\right)<\mathrm{d}_{\mathrm{T}}\left(y_{2}, x_{1}\right)$. Now $\mathrm{d}_{\mathrm{T}}\left(\bar{h} \cdot p, \bar{h} \cdot x_{1}\right)=\mathrm{d}_{\mathrm{T}}\left(\bar{h} \cdot p, x_{1}\right)$ and $\bar{h} \cdot p$ is also on the arc. $\bar{h} \cdot p$ cannot be on the open segment between $p$ and $x_{1}$. If $\bar{h} \cdot p$ were on the open segment between $p$ and $y_{2}$, the Tits geodesic from $\bar{h} \cdot p$ to $x_{1}$ would go through $p$ or $y_{2}$, both would contradict $\mathrm{d}_{\mathrm{T}}\left(\bar{h} \cdot p, x_{1}\right)=\mathrm{d}_{\mathrm{T}}\left(p, x_{1}\right)$. So $\bar{h} \cdot p=p$. Then $p \in \partial \operatorname{Min}(h)$ and lies on a path in $\partial \operatorname{Min}(h)$ joining $h^{ \pm \infty}$, forcing the arc to be in $\partial \operatorname{Min}(h)$, which contradicts the previous assertion.

We describe our strategy for proving the main result about endpoints of hyperbolic elements in this section: Denote the segment in $\partial X$ from $g^{\infty}$ to $z$ passing through $g^{-\infty}$ by $\beta$. Let $y$ be the point where $\beta$ intersects $C_{2}$. The essence of the following arguments is to look for a point in $\partial_{\mathrm{T}} X$ that is over $\pi / 2$ away from $C_{1}$ or $C_{2}$, which are closed $G$-invariant subsets, so obtaining a contradiction to Theorem 3.1.

Lemma 3.4 $g^{-\infty}$ cannot be on the closed segment in $\beta$ from $g^{\infty}$ to $y$. 
Proof Suppose $g^{-\infty}$ is on that segment. Since $\mathrm{d}_{\mathrm{T}}\left(g^{\infty}, g^{-\infty}\right)=\pi$, the Tits length of this segment from $g^{\infty}$ to $y$ is at least $\pi$. Let $0<\delta<\pi / 2$ be such that $2 \delta \leq \mathrm{d}_{\mathrm{T}}\left(y, C_{1}\right)$. Take a point $p$ on this segment so that $\mathrm{d}_{\mathrm{T}}\left(p, g^{\infty}\right)=\pi / 2+\delta$. Then $\mathrm{d}_{\mathrm{T}}(p, y) \geq \pi / 2-\delta$. Now for any point $x \in C_{1}$ other than $g^{\infty}$, if the Tits geodesic segment from $p$ to $x$ passes through $y$, then

$$
\mathrm{d}_{\mathrm{T}}(p, x) \geq \mathrm{d}_{\mathrm{T}}(p, y)+\mathrm{d}_{\mathrm{T}}\left(y, C_{1}\right) \geq(\pi / 2-\delta)+2 \delta=\pi / 2+\delta ;
$$

while if it passes through $g^{\infty}$, then obviously $\mathrm{d}_{\mathrm{T}}(p, x)>\mathrm{d}_{\mathrm{T}}\left(p, g^{\infty}\right)=\pi / 2+\delta$. So $\mathrm{d}_{\mathrm{T}}\left(p, C_{1}\right) \geq \pi / 2+\delta$, which contradicts Theorem 3.1.

Now we deal with the case that $g^{-\infty}$ is in the open segment in $\beta$ from $y$ to $z$. We state a lemma first which will also be used in later arguments.

Lemma 3.5 Suppose $h \in G$ is a hyperbolic element such that $F_{0} \subset \operatorname{Min}(h)$ whose boundary intersects $C_{1}$ and $C_{2}$ alternatively at $x_{1}, y_{1}, x_{2}, y_{2}$. Assume that the endpoint $h^{-\infty}$ is on some open arc, say the open arc between $x_{i}$ and $y_{j}$, while another endpoint $h^{\infty}$ is not contained in the closed arc between $x_{i}$ and $y_{j}$. Then for any point $x \in C_{1}$ other than $x_{1}$ and $x_{2}$, the sequence $\bar{h}^{n} \cdot x$ can only accumulate at $x_{1}$ or $x_{2}$. Similarly, for any point $y \in C_{2}$ other than $y_{1}$ and $y_{2}$, the sequence $\bar{h}^{n} \cdot x$ can only accumulate at $y_{1}$ or $y_{2}$.

Proof Suppose not, then the sequence has an accumulation point $x^{\prime} \in C_{1} \backslash\left\{x_{1}, x_{2}\right\}$. By Lemma 2.2, $x^{\prime}$ forms boundary of a half flat plane with $h^{ \pm \infty}$. This boundary goes from $h^{\infty}$ to $x^{\prime}$, and then passes through $x_{i}$ or $y_{j}$ before ending at $h^{-\infty}$. If it passes through $x_{i}$, then the Tits length of segment on this boundary joining $h^{\infty}$ to $x_{i}$ is the total length of the half-plane boundary $\pi$ minus the length of the segment from $x_{i}$ to $h^{-\infty}$, thus it is equal to the length of the Tits geodesic segment on $\partial F_{0}$ joining these two points, so there are two geodesics for these two points. But this contradicts the uniqueness of Tits geodesic between two points less than $\pi$ apart. If the boundary of the half flat plane goes through $y_{j}$, apply the same argument to the points $h^{\infty}$ and $y_{j}$ and we have the same contradiction. For the case $y \in C_{2} \backslash\left\{y_{1}, y_{2}\right\}$ use the same argument.

Lemma 3.6 $g^{-\infty}$ cannot be in the open segment from $y$ to $z$.

Proof Suppose $g^{-\infty}$ is on this segment. For any point $z^{\prime} \in C_{1}$ other than $g^{\infty}$ and $z$, the sequence $\bar{g}^{-n} \cdot z^{\prime}$ converges to $z$ by Lemma 3.5 and Lemma 2.2 which says that $\bar{g}^{-n} \cdot z^{\prime}$ cannot accumulate at $g^{\infty}$. 
The segment $\beta$ has Tits length larger than $\pi$, so there is a point $w \in \beta$ which is more than $\pi / 2$ away from $g^{\infty}$ and from $z$.

By lower semi-continuity of the Tits metric,

$$
\begin{aligned}
\mathrm{d}_{\mathrm{T}}\left(w, z^{\prime}\right) & =\lim _{n \rightarrow \infty} \mathrm{d}_{\mathrm{T}}\left(\bar{g}^{-n} \cdot w, \bar{g}^{-n} \cdot z^{\prime}\right) \\
& \geq \mathrm{d}_{\mathrm{T}}\left(\lim _{n \rightarrow \infty} \bar{g}^{-n} \cdot w, \lim _{n \rightarrow \infty} \bar{g}^{-n} \cdot z^{\prime}\right)=\mathrm{d}_{\mathrm{T}}(w, z) .
\end{aligned}
$$

So $\mathrm{d}_{\mathrm{T}}\left(w, C_{1}\right)>\pi / 2$, a contradiction to Theorem 3.1.
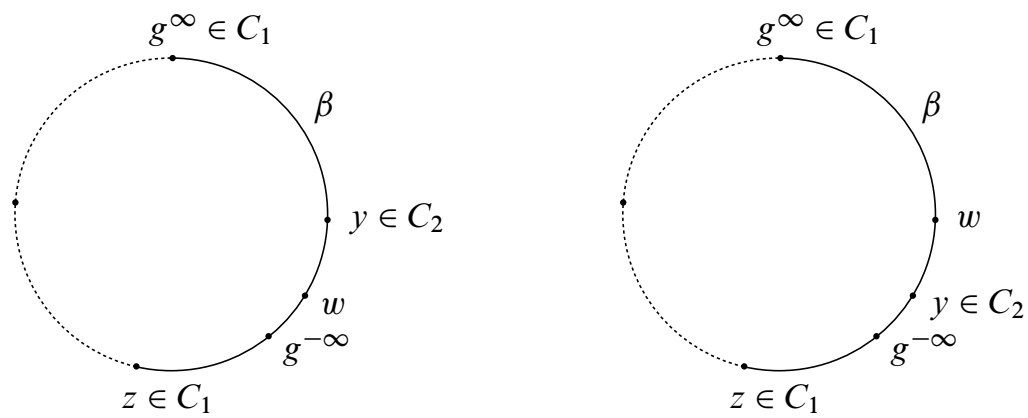

Figure 2: $\partial F$ in Lemma 3.6

We see from these lemmas that the endpoints of a hyperbolic element must be both in $C_{1}$, or both in $C_{2}$, or none is in $C_{1} \cup C_{2}$.

If $g$ is a hyperbolic element of $G$ with endpoints not in $C_{1} \cup C_{2}$, we have the following results.

Lemma 3.7 $\partial \operatorname{Min}(g)$ is the boundary of a 2-flat.

Proof Since $\partial \operatorname{Min}(g)$ is a suspension, so it can only be a circle or a set of two points. However, as $\bar{g}$ is an isometry of $\partial_{\mathrm{T}} X$, we see that $\bar{g}$ must fix the arc on which $g^{\infty}$ lies. So $\partial \operatorname{Min}(g)=\operatorname{Fix}(\bar{g})$ can only be a circle. Then by the same reason as in Lemma 3.2 $\operatorname{Min}(g)$ contains a $2-$ flat, whose boundary is the circle.

Suppose for convenience that $g^{\infty}$ is on the open arc from $x_{1} \in C_{1}$ to $y_{1} \in C_{2}$, and $x_{2} \in C_{1}, y_{2} \in C_{2}$ are the two other points on the boundary $\partial F$.

Lemma 3.8 For $g$ as above, $g^{-\infty}$ can only be on the open arc from $x_{2}$ to $y_{2}$. 
Proof Suppose $g^{-\infty}$ were not on this arc. Without loss of generality let $g^{-\infty}$ be on the arc joining $y_{1}$ and $x_{2}$. Now the segment from $x_{1}$ to $x_{2}$ through $y_{1}$ has Tits length larger than $\pi$, so we can choose a point $p$ on this segment so that $p$ is at distance more than $\pi / 2$ away from $x_{1}$ and $x_{2}$. By Lemma 3.5, for any other point $x^{\prime} \in C_{1}, \bar{g}^{n} \cdot x^{\prime}$ cannot have an accumulation point other than $x_{1}$ and $x_{2}$. Passing to a subsequence $\bar{g}^{n_{k}} \cdot x^{\prime} \rightarrow x_{i}, i=1$ or 2 , we have

$$
\begin{aligned}
\mathrm{d}_{\mathrm{T}}\left(p, x^{\prime}\right) & =\lim _{n_{k} \rightarrow \infty} \mathrm{d}_{\mathrm{T}}\left(\bar{g}^{n_{k}} \cdot p, \bar{g}^{n_{k}} \cdot x^{\prime}\right) \\
& \geq \mathrm{d}_{\mathrm{T}}\left(\lim _{n_{k} \rightarrow \infty} \bar{g}^{n_{k}} \cdot p, \lim _{n_{k} \rightarrow \infty} \bar{g}^{n_{k}} \cdot x^{\prime}\right)=\mathrm{d}_{\mathrm{T}}\left(p, x_{i}\right),
\end{aligned}
$$

then $\mathrm{d}_{\mathrm{T}}\left(p, C_{1}\right)>\pi / 2$, contradicting Theorem 3.1.

\section{Main result}

Now we add the assumption that $G$ contains a subgroup isomorphic to $\mathbb{Z}^{2}$, then the flat torus theorem [3, Theorem II.7.1] implies that there exist two commuting hyperbolic elements $g_{1}, g_{2} \in G$, such that $\operatorname{Min}\left(g_{1}\right)$, formed by the axes of $g_{1}$, contains axes of $g_{2}$ not parallel to those of $g_{1}$. Then an axis of $g_{1}$ and an axis of $g_{2}$ span a 2-flat in $\operatorname{Min}\left(g_{1}\right)$, and elements $g_{1}^{n} g_{2}^{m}$ are also hyperbolic and have axes in this 2-flat with endpoints dense on the boundary of this $2-$ flat. So we can choose some hyperbolic element $g$ so that its endpoints are not in $C_{1} \cup C_{2}$.

We start with a lemma about the orbits of the group action, then we will prove Theorem 1.1.

Lemma 4.1 For any two distinct points $w_{1}, w_{2} \in \partial X$, there is a sequence $\left(g_{i}\right)_{i=0}^{\infty} \subset G$ such that the points $\bar{g}_{i} \cdot w_{j}$, where $0 \leq i<\infty$ and $j \in\{1,2\}$, are distinct.

Proof From Lemma 2.4 we know that every $w \in \partial X$ has an infinite orbit $G \cdot w$. So let $\left(h_{i}\right)_{i=0}^{\infty} \subset G$ be a sequence such that $\bar{h}_{i} \cdot w_{1}$ are distinct. We will construct the sequence $\left(g_{i}\right)$ inductively. First set $g_{0}=e$.

Suppose that for $n \geq 0$ we have $g_{0}, \ldots, g_{n}$ such that $\bar{g}_{i} \cdot w_{j}$, where $0 \leq i \leq n$ and $j \in\{1,2\}$, are distinct. Let $S_{n}:=\left\{\bar{g}_{m} \cdot w_{1}, \bar{g}_{m} \cdot w_{2}: 0 \leq m \leq n\right\}$. Pass to a subsequence of $\left(h_{i}\right)$ so that $\bar{h}_{i} \cdot w_{1} \notin S_{n}$. (We will keep denoting any subsequence by $\left(h_{i}\right)$.) If there exists some $h_{j}$ such that $\bar{h}_{j} \cdot w_{2} \notin S_{n}$, then set $g_{n+1}=h_{j}$. Otherwise, there exists some $\bar{g}_{m} \cdot w_{k} \in S_{n}$ such that $\bar{h}_{i} \cdot w_{2}=\bar{g}_{m} \cdot w_{k}$ for infinitely many $h_{i}$. Pass to this subsequence. Since the orbit of $\bar{g}_{m} \cdot w_{k}$ is infinite, there exists $h^{\prime} \in G$ such that $\overline{h^{\prime}} \cdot\left(\bar{g}_{m} \cdot w_{k}\right) \notin S_{n}$, so $\overline{h^{\prime} h_{i}} \cdot w_{2} \notin S_{n}$. Now $\overline{h^{\prime} h_{i}} \cdot w_{1} \notin S_{n}$ for infinitely many $h_{i}$. Set $g_{n+1}=h^{\prime} h_{i}$ for one such $h_{i}$. Hence we get the desired sequence $\left(g_{i}\right)$. 
Remark The only condition required on the group action is that every orbit is infinite. This proof can be used to show a similar result for any finite set $\left\{w_{1}, \ldots w_{n}\right\}$.

Lemma 4.2 For any $x \in C_{1}, y \in C_{2}$ we have $\mathrm{d}_{\mathrm{T}}(x, y)=\pi / 2$. Hence $\partial_{\mathrm{T}} X$ is metrically a spherical join of $C_{1}$ and $C_{2}$.

Proof Consider some $g \in G$ which is hyperbolic with endpoints not on $C_{1} \cup C_{2}$. Let $\partial \operatorname{Min}(g)=\partial F$. We will first prove that for $x_{1}, x_{2} \in C_{1} \cap \partial F, y_{1}, y_{2} \in C_{2} \cap \partial F$, we have $\mathrm{d}_{\mathrm{T}}\left(x_{i}, y_{j}\right)=\pi / 2$, where $i, j=1,2$. Take any of the four arcs making up $\partial F$, say the arc joining $x_{1}$ and $y_{1}$.

The endpoints of hyperbolic elements in $Z_{g}$ are dense on $\partial F$, so we can pick a $g^{\prime} \in Z_{g}$ so that $g^{\prime-\infty}$ is as close to the midpoint of arc $x_{2}$ and $y_{2}$ as we want. Let $0<\delta<$ $\min \left(\mathrm{d}_{\mathrm{T}}\left(x_{2}, C_{2}\right), \mathrm{d}_{\mathrm{T}}\left(y_{2}, C_{1}\right)\right)$. Pick $g^{\prime}$ so that $\left|\mathrm{d}_{\mathrm{T}}\left(g^{\prime-\infty}, x_{2}\right)-\mathrm{d}_{\mathrm{T}}\left(g^{\prime-\infty}, y_{2}\right)\right|<\delta$. For any point $x \in C_{1}$ other than $x_{2}$, if the Tits geodesic segment from $g^{\prime-\infty}$ to $x$ passes through $y_{2}$, then

$$
\begin{aligned}
\mathrm{d}_{\mathrm{T}}\left(g^{\prime-\infty}, x\right) & \geq \mathrm{d}_{\mathrm{T}}\left(g^{\prime-\infty}, y_{2}\right)+\mathrm{d}_{\mathrm{T}}\left(y_{2}, C_{1}\right) \\
& >\mathrm{d}_{\mathrm{T}}\left(g^{\prime-\infty}, x_{2}\right)-\delta+\mathrm{d}_{\mathrm{T}}\left(y_{2}, C_{1}\right)>\mathrm{d}_{\mathrm{T}}\left(g^{\prime-\infty}, x_{2}\right) ;
\end{aligned}
$$

while if it passes through $x_{2}$ then obviously $\mathrm{d}_{\mathrm{T}}\left(g^{\prime-\infty}, x\right)>\mathrm{d}_{\mathrm{T}}\left(g^{\prime-\infty}, x_{2}\right)$. For any $y \in C_{2}$ other than $y_{2}$, by similar reasoning on the Tits geodesic segment from $g^{\prime-\infty}$ to $y$, we have $\mathrm{d}_{\mathrm{T}}\left(g^{\prime-\infty}, y\right)>\mathrm{d}_{\mathrm{T}}\left(g^{\prime-\infty}, y_{2}\right)$.

For any arc joining $x \neq x_{2} \in C_{1}$ and $y \neq y_{2} \in C_{2}$, since $\mathrm{d}_{\mathrm{T}}\left(g^{\prime-\infty}, x\right)>\mathrm{d}_{\mathrm{T}}\left(g^{\prime-\infty}, x_{2}\right)$, the point $x_{2}$ cannot be an accumulation point of ${\overline{g^{\prime}}}^{n} \cdot x$ by Lemma 2.2, then by Lemma $3.5, \bar{g}^{\prime}{ }^{n} \cdot x \rightarrow x_{1}$. Likewise, ${\overline{g^{\prime}}}^{n} \cdot y \rightarrow y_{1}$. So

$$
\begin{aligned}
\mathrm{d}_{\mathrm{T}}(x, y) & =\lim _{n \rightarrow \infty} \mathrm{d}_{\mathrm{T}}\left({\overline{g^{\prime}}}^{n} \cdot x, \bar{g}^{\prime} \cdot y\right) \\
& \geq \mathrm{d}_{\mathrm{T}}\left(\lim _{n \rightarrow \infty}{\overline{g^{\prime}}}^{n} \cdot x, \lim _{n \rightarrow \infty}{\overline{g^{\prime}}}^{n} \cdot y\right) \\
& =\mathrm{d}_{\mathrm{T}}\left(x_{1}, y_{1}\right) .
\end{aligned}
$$

For any other arc joining $x_{i}$ to $y_{j}$ in $\partial F$, by Lemma 4.1 there exists $h \in G$ such that $\bar{h} \cdot x_{i} \neq x_{2}$ and $\bar{h} \cdot y_{j} \neq y_{2}$, so from the inequality (4-1) we get

$$
\mathrm{d}_{\mathrm{T}}\left(x_{i}, y_{j}\right)=\mathrm{d}_{\mathrm{T}}\left(\bar{h} \cdot x_{i}, \bar{h} \cdot y_{j}\right) \geq \mathrm{d}_{\mathrm{T}}\left(x_{1}, y_{1}\right) .
$$

Thus all arcs have equal length $\pi / 2$. Now for any $x \in C_{1}, y \in C_{2}$, by Lemma 3.5 the sequence $\bar{g}^{n} \cdot x$ can accumulate at $x_{1}$ or $x_{2}$, and $\bar{g}^{n} \cdot y$ can accumulate at $y_{1}$ or $y_{2}$, so passing to some subsequence $\left(\bar{g}^{n_{k}}\right)$, we have convergence sequences $\bar{g}^{n_{k}} \cdot x \rightarrow x_{i}$ 
and $\bar{g}^{n_{k}} \cdot y \rightarrow y_{j}$. Then we have the inequality

$$
\mathrm{d}_{\mathrm{T}}(x, y)=\lim _{n_{k} \rightarrow \infty} \mathrm{d}_{\mathrm{T}}\left(\bar{g}^{n_{k}} \cdot x, \bar{g}^{n_{k}} \cdot y\right) \geq \mathrm{d}_{\mathrm{T}}\left(x_{i}, y_{j}\right)=\pi / 2 .
$$

Take a point $p$ on the open arc joining $x$ and $y$. Without loss of generality assume that $p$ and $x$ are connected in $\partial_{\mathrm{T}} X$ by a segment in the arc. For any $\epsilon>0$, we may choose a new point on the segment from $p$ to $x$ to replace $p$ so that $0<\mathrm{d}_{\mathrm{T}}(x, p)<\epsilon$. Consider the Tits geodesic from $p$ to some point in $C_{2}$. If it passes through $x$, then it consists of the segment from $p$ to $x$ and an arc from $x$ to some point in $C_{2}$, so by the inequality (4-2) its Tits length is at least $\pi / 2+\mathrm{d}_{\mathrm{T}}(x, p)$. By Theorem 3.1 $\mathrm{d}_{\mathrm{T}}\left(p, C_{2}\right) \leq \pi / 2$, so there must be a Tits geodesic from $p$ to some point in $C_{2}$ that does not pass through $x$, hence it passes through $y$. Its length is at least $\mathrm{d}_{\mathrm{T}}(p, y)$, so $y$ is the closest point in $C_{2}$ to $p$, so $\mathrm{d}_{\mathrm{T}}(p, y)=\mathrm{d}_{\mathrm{T}}\left(p, C_{2}\right) \leq \pi / 2$. Then $\mathrm{d}_{\mathrm{T}}(x, y) \leq$ $\mathrm{d}_{\mathrm{T}}(x, p)+\mathrm{d}_{\mathrm{T}}(p, y)<\pi / 2+\epsilon$. Letting $\epsilon \rightarrow 0$ we have $\mathrm{d}_{\mathrm{T}}(x, y) \leq \pi / 2$. Combining with the inequality (4-2), $\mathrm{d}_{\mathrm{T}}(x, y)=\pi / 2$.

Theorem 4.3 If $X$ is a CAT(0) space which admits a geometric group action by a group containing a subgroup isomorphic to $\mathbb{Z}^{2}$, and $\partial X$ is homeomorphic to the join of two Cantor sets, then $\partial_{\mathrm{T}} X$ is the spherical join of two uncountable discrete sets. If $X$ is geodesically complete, that is, every geodesic segment in $X$ can be extended to a geodesic line, then $X$ is a product of two CAT(0) space $X_{1}, X_{2}$ with $\partial X_{i}$ homeomorphic to a Cantor set.

Proof We have shown that for any $x \in C_{1}, y \in C_{2}, \mathrm{~d}_{\mathrm{T}}(x, y)=\pi / 2$ in Lemma 4.2, so every two distinct points in $C_{i}$ has Tits distance at most $\pi$ for $i=1,2$. Since the identity map $\partial_{\mathrm{T}} X \rightarrow \partial X$ is continuous, any Tits path joining two distinct points in $C_{i}$ is also a path in the visual boundary $\partial X$, and every such path in $\partial X$ has to pass through some point in the other $C_{j}$, thus the distance between the two point must be exactly $\pi$, hence $C_{i}$ with the Tits metric is an uncountable discrete set. Then $\partial_{\mathrm{T}} X$ is isomorphic to the spherical join of $C_{1}$ and $C_{2}$, giving the first result. So with the additional assumption that $X$ is geodesically complete, it follows by BridsonHaefliger [3, Theorem II.9.24] that $X$ splits as a product $X_{1} \times X_{2}$, with $\partial X_{i}=C_{i}$ for $i=1,2$.

\section{Some properties of the group}

We will show Theorem 1.2 in this section. Assuming that $X$ is geodesically complete, and hence reducible by Theorem 4.3, we have the following result for the group $G$. We do not require that $G$ stabilizes each of $C_{1}$ and $C_{2}$ in this section. 
Theorem 5.1 Let $X$ be a CAT(0) space such that $\partial X$ is homeomorphic to the join of two Cantor sets and suppose $X$ is geodesically complete. For a group $G<\operatorname{Isom}(X)$ containing $\mathbb{Z}^{2}$ and acting geometrically on $X$, either $G$ or a subgroup of it of index 2 is a uniform lattice in $\operatorname{Isom}\left(X_{1}\right) \times \operatorname{Isom}\left(X_{2}\right)$, where $X_{1}, X_{2}$ are given by Theorem 4.3

Proof We know from Theorem 4.3 that $X=X_{1} \times X_{2}$, so we only need to show that $G$ or a subgroup of it of index 2 preserves this decomposition.

By Lemma 2.1, either $G$ or a subgroup of it of index 2 stabilizes $C_{1}$ and $C_{2}$. Replacing $G$ by its subgroup if necessary, we assume $G$ stabilizes $C_{1}$ and $C_{2}$.

Denote by $\pi_{i}$ the projection of $X$ to $X_{i}, i=1,2$. Take any $p_{1}, p_{2} \in X$ such that $\pi_{2}\left(p_{1}\right)=\pi_{2}\left(p_{2}\right)$. Extend $\left[p_{1}, p_{2}\right]$ to a geodesic line $\gamma$, its projection to each of $X_{i}$ is the image of a geodesic line. Since $X_{1}$ is totally geodesic, the geodesic segment $\left[p_{1}, p_{2}\right]$ projects to a single point $\pi_{2}\left(p_{1}\right)$ on $X_{2}$, that is, a degenerated geodesic segment, so $\pi_{2}(\gamma)$ is also a degenerated geodesic line. Thus the endpoints $\gamma( \pm \infty)$ are in $C_{1}$. Now $g \cdot \gamma$ is a geodesic line passing through $g \cdot p_{1}, g \cdot p_{2}$, and its endpoints $\bar{g} \cdot \gamma( \pm \infty) \in C_{1}$, so $\pi_{2}\left(g \cdot p_{1}\right)=\pi_{2}\left(g \cdot p_{2}\right)$. Similarly, for any $q_{1}, q_{2} \in X$ such that $\pi_{1}\left(q_{1}\right)=\pi_{1}\left(q_{2}\right)$ we have $\pi_{1}\left(g \cdot q_{1}\right)=\pi_{1}\left(g \cdot q_{2}\right)$. So $G$ preserves the decomposition $X=X_{1} \times X_{2}$, hence the result.

We will show that $\operatorname{Isom}\left(X_{i}\right)$ is isomorphic to a subgroup of $\operatorname{Homeo}\left(C_{i}\right)$ by the following lemma.

Lemma 5.2 Suppose $X^{\prime}$ is a proper complete CAT(0) space, and $G^{\prime}<\operatorname{Isom}\left(X^{\prime}\right)$ acts properly on $X^{\prime}$ by isometries.

(1) If $S \subset \partial X^{\prime}$ is a set of points on the boundary such that the intersection

$$
\bigcap_{w \in S} \overline{\mathrm{B}_{\mathrm{T}}(w, \pi / 2)}
$$

is empty, then there exists a point $q \in X$ such that any $g \in \operatorname{Isom}\left(X^{\prime}\right)$ that stabilizes all horospheres with centers in $S$ will fix $q$. In particular, such $g$ is elliptic.

(2) Assume that $G^{\prime}$ does not have parabolic isometries of positive translation lengths. If $\partial X^{\prime}$ is not a suspension and the radius of $\partial_{\mathrm{T}} X^{\prime}$ is larger than $\pi / 2$, then the map $G^{\prime} \rightarrow \operatorname{Homeo}\left(\partial X^{\prime}\right)$, defined by extending the action of $G^{\prime}$ to the boundary $\partial X^{\prime}$, has a finite kernel, that is, the subgroup of $G^{\prime}$ that acts trivially on the boundary is finite. Moreover, assume the action of $G^{\prime}$ is cocompact, then the kernel fixes a subspace of $X^{\prime}$ with boundary $\partial X^{\prime}$. 
Proof To prove (1), as any such $g$ stabilizes all horospheres by assumption, thus $g$ stabilizes all horoballs centered at every $w \in S$. Take an arbitrary point $q^{\prime} \in X$ and choose for each $w$ a closed horoball $H_{w}$ centered at $w$ that contains $q^{\prime}$. Their intersection $\bigcap_{w \in S} H_{w}$ is non-empty since it contains $q^{\prime}$. By Caprace-Monod [6, Lemma 3.5] $\partial H_{w}=\overline{\mathrm{B}_{\mathrm{T}}(w, \pi / 2)}$, then $\partial\left(\bigcap_{w \in S} H_{w}\right) \subset \bigcap_{w \in S}\left(\partial H_{w}\right)=\varnothing$. So $\bigcap_{w \in S} H_{w}$ is bounded. Also as every $H_{w}$ is stabilized by $g$, so is $\bigcap_{w \in S} H_{w}$. As $\bigcap_{w \in S} H_{w}$ is convex and compact, it contains a unique circumcenter $q$. Then $g$ fixes $q$.

To prove (2), first we claim that if $g \in G^{\prime}$ has zero translation length, and $g$ fixes a point $w \in \partial X$, then the horospheres centered at $w$ are stabilized by $g$. Let $\gamma$ be a geodesic ray with endpoint $w$, and $b_{\gamma}(\cdot)$ be the corresponding Busemann function. Since $g \cdot \gamma$ is asymptotic to $\gamma$, we have $b_{\gamma}(x)=b_{g \cdot \gamma}(g \cdot x)=b_{\gamma}(g \cdot x)+C$ for some constant $C$. Then as Busemann functions are 1-Lipschitz, it follows that $|C| \leq \mathrm{d}_{X}(x, g \cdot x)$. We have assumed that $|g|=\inf _{x} \mathrm{~d}_{X}(x, g \cdot x)=0$, so $C=0$, that is, $b_{\gamma}(x)=b_{\gamma}(g \cdot x)$, hence the claim.

Now if $g \in G^{\prime}$ acts by hyperbolic isometry, then $\partial \operatorname{Min}(g)=\operatorname{Fix}(\bar{g})$ is a suspension. Since we assumed $\partial X^{\prime}$ is not a suspension, any $g$ acting trivially on the whole boundary $\partial X^{\prime}$ is not hyperbolic, so by assumption $g$ is either elliptic or parabolic with zero translation length, thus by the previous claim $g$ stabilizes all the horospheres centered at any point on $\partial X^{\prime}$. As $\partial_{\mathrm{T}} X^{\prime}$ has radius larger than $\pi / 2$, for every $x \in \partial X^{\prime}$ there is some $w \in \partial X^{\prime}$ such that $\mathrm{d}_{\mathrm{T}}(x, w)>\pi / 2$, so $x \notin \overline{\mathrm{B}_{\mathrm{T}}(w, \pi / 2)}$, hence $S=\partial X^{\prime}$ satisfies the condition in (1). Now (1) implies that the kernel of $G^{\prime} \rightarrow \operatorname{Homeo}\left(\partial X^{\prime}\right)$ is a subgroup of the stabilizer of some point $q \in X^{\prime}$. As the action of $G^{\prime}$ is proper, the kernel is finite.

Let $K$ be the kernel. The set fixed by $K$ is closed and convex. For any point $q$ fixed by the kernel, as $g \cdot q$ is fixed by $g K^{-1}=K$, then $G^{\prime} \cdot q$ is fixed by $K$. If the action of $G^{\prime}$ is cocompact, then $G^{\prime} \cdot q$ has boundary $\partial X^{\prime}$, and thus so is the set fixed by $K$.

Remark If we further assume that the space of directions at any point of $X^{\prime}$ is compact (for instance, when $X^{\prime}$ is geodesically complete), then it was proved by Fujiwara, Nagano and Shioya [7] that the fixed point set on $\partial_{\mathrm{T}} X^{\prime}$ of any parabolic isometry, possibly with positive translation length, has Tits radius $\leq \pi / 2$. So in this case the assumption on parabolic isometries in (2) of the previous lemma is not needed.

Corollary 5.3 Let $X$ be a geodesically complete CAT(0) space such that $\partial X$ is homeomorphic to the join of two Cantor sets. Then for a group $G<\operatorname{Isom}(X)$ containing $\mathbb{Z}^{2}$ and acting geometrically on $X$, either $G$ or a subgroup of it of index 2 is isomorphic to a subgroup of $\operatorname{Homeo}\left(C_{1}\right) \times \operatorname{Homeo}\left(C_{2}\right)$.

Proof This follows from Theorem 5.1 and Lemma 5.2. 
We can still show this without the geodesic completeness assumption.

Theorem 5.4 Let $X$ be a CAT(0) space such that $\partial X$ is homeomorphic to the join of two Cantor sets. Then for a group $G<\operatorname{Isom}(X)$ containing $\mathbb{Z}^{2}$ and acting geometrically on $X$, a finite quotient of either $G$ or a subgroup of $G$ of index 2 is isomorphic to a subgroup in $\operatorname{Homeo}\left(C_{1}\right) \times \operatorname{Homeo}\left(C_{2}\right)$.

Proof Assume $G$ stabilizes each of $C_{1}$ and $C_{2}$ as in the proof of Theorem 5.1. Each $g \in G$ acts on $\partial X$ as a homeomorphism, so it acts on $C_{i} \subset \partial X$ also as a homeomorphism.

Suppose $\bar{g}$ acts trivially on $C_{1}$ and $C_{2}$, that is, $g$ is in the kernel of $G \rightarrow \operatorname{Homeo}\left(C_{1}\right) \times$ Homeo $\left(C_{2}\right)$. Then for any point $x \in \partial X$ outside $C_{1} \cup C_{2}$, the arc on which $x$ lies is a Tits geodesic segment of length $\pi / 2$ in $\partial_{\mathrm{T}} X$. Since $\bar{g}$ acts on $\partial_{\mathrm{T}} X$ by isometry and both endpoints of this Tits geodesic segment are fixed by $\bar{g}$, so $\bar{g}$ fixes the whole arc, thus $\bar{g} \cdot x=x$. Hence $\bar{g}$ acts trivially on $\partial X$. One can check that $\partial_{\mathrm{T}} X$ has radius larger than $\pi / 2$, so by Lemma $5.2 G \rightarrow \operatorname{Homeo}(\partial X)$ has finite kernel. Hence the result.

In the case when $X$ is geodesically complete, actually we can prove a stronger result, expressed in the last statement of Theorem 1.2. Observe that $X_{i}$ is a Gromov hyperbolic space by the flat plane theorem, which states that a proper cocompact CAT(0) space $Y$ is hyperbolic if and only if it does not contain a subspace isometric to $\mathbb{E}^{2}$. Recall that a cocompact space is defined as a space $Y$ which has a compact subset whose images under the action by $\operatorname{Isom}(Y)$ cover $Y$. The (projected) action of $G$ on $X_{i}$ is cocompact, even though the image in $\operatorname{Isom}\left(X_{i}\right)$ may not be discrete. As $\partial X_{i}$ does not contain $S^{1}$, the result follows.

We will show $X_{i}$ is quasi-isometric to a tree. This is equivalent to having the bottleneck property by a theorem of Manning, which he proved with an explicit construction:

Theorem 5.5 (Manning [9, Theorem 4.6]) Let $Y$ be a geodesic metric space. The following are equivalent:

(1) $Y$ is quasi-isometric to some simplicial tree $\Gamma$.

(2) (Bottleneck property) There is some $\Delta>0$ so that for all $x, y \in Y$ there is a midpoint $m=m(x, y)$ with $d(x, m)=d(y, m)=\frac{1}{2} d(x, y)$ and the property that any path from $x$ to $y$ must pass within less than $\Delta$ of the point $m$.

Pick a base point $p$ in $X_{i}$. There exists some $r>0$ such that $G \cdot B(p, r)$ covers $X_{i}$.

Lemma 5.6 There exists $R>0$ such that for any $x, y$ in the same connected component of $X_{i} \backslash B(p, R)$, the geodesic segment $[x, y]$ does not intersect $B(p, r)$. 
Proof Suppose on the contrary that for $R_{n}$ increasing to infinity, we can find $x_{n}, y_{n}$ in the same connected component of $X_{i} \backslash B\left(p, R_{n}\right)$ and $\left[x_{n}, y_{n}\right]$ intersects $B(p, r)$. Since $\bar{X}_{i}$ is compact in the cone topology, passing to a subsequence we have $x_{n} \rightarrow \bar{x}$, $y_{n} \rightarrow \bar{y}$ for some $\bar{x}, \bar{y} \in \partial X_{i}$. By Bridson-Haefliger [3, Lemma II.9.22], there is a geodesic line from $\bar{x}$ to $\bar{y}$ intersecting $B(p, r)$. In particular, $\bar{x} \neq \bar{y}$.

Since different connected components in the boundary of a hyperbolic space correspond to different ends of the space (see Bridson-Haefliger [3, Exercise III.H.3.8]), and $\partial X_{i}$ is a Cantor set, so $\bar{x}$ and $\bar{y}$ are in different ends of $X_{i}$, which are separated by $B\left(p, R_{n}\right)$ for $R_{n}$ large enough. But then $x_{n}, y_{n}$ will be in different connected components of $X_{i} \backslash B\left(p, R_{n}\right)$, contradicting the assumption. Hence the result.

\section{Lemma 5.7 $X_{i}$ has the bottleneck property.}

Proof For any $x, y \in X_{i}$, we may translate by some $g \in G$ so that the midpoint $m$ of $[x, y]$ is in $B(p, r)$. We may assume that $d(x, y)>2(R+r)$, then $x, y \in X_{i} \backslash B(p, R)$. By Lemma 5.6, $x, y$ are in different connected components of $X_{i} \backslash B(p, R)$, hence any path connecting $x$ to $y$ must intersect $B(p, R)$, so some point on this path is at a distance at most $R+r$ from $m$. Thus the bottleneck property is satisfied.

Lemma 5.8 $X_{i}$ is quasi-isometric to a bounded valence tree with no terminal vertex.

Proof First we describe briefly Manning's construction in his proof of Theorem 5.5. Let $R^{\prime}=20 \Delta$. Start with a single point $\star$ in $Y$. Call the vertex set containing this point $V_{0}$, and let $\Gamma_{0}$ be a tree with only one vertex and no edge, and $\beta_{0}: \Gamma_{0} \rightarrow Y$ be the map sending the vertex to $\star$. Then for each $k \geq 1$, Let $N_{k-1}$ be the open $R$-neighborhood of $V_{k-1}$. Let $\mathcal{C}_{k}$ be the set consists of path components of $Y \backslash N_{k-1}$. For each $C \in \mathcal{C}_{k}$ pick some point $v$ at $C \cap \bar{N}_{k}$. There is a unique path component in $\mathcal{C}_{k-1}$ containing $C$, corresponding to a terminal vertex $w \in V_{k-1}$. Connect $v$ to $w$ by a geodesic segment. Let $V_{k}$ be the union of $V_{k-1}$ and the set of new points from each of the path components in $\mathcal{C}_{k}$. Add new vertices and edges to the tree $\Gamma_{k-1}$ accordingly to get the tree $\Gamma_{k}$. Extend $\beta_{k-1}$ to $\beta_{k}$ by mapping new vertices of $\Gamma_{k}$ to corresponding new vertices in $V_{k}$, and new edges to corresponding geodesic segments. The tree $\Gamma=\bigcup_{k \geq 0} \Gamma_{k}$, and $\beta: \Gamma \rightarrow Y$ is defined to be $\beta_{k}$ on $\Gamma_{k}$.

Apply the construction above to $X_{i}$. Since $X_{i}$ is geodesically complete, each terminal vertex in $V_{k-1}$ will be connected by at least one vertex in $V_{k} \backslash V_{k-1}$, and similarly so for terminal vertices of $\Gamma_{k-1}$. So the tree $\Gamma$ has no terminal vertex.

Manning proved that the length of each geodesic segment added in the construction is bounded above by $R^{\prime}+6 \Delta$. Consider $w \in V_{k-1}$ with corresponding path component 
$C_{w} \in \mathcal{C}_{k-1}$. Every path component $C \in \mathcal{C}_{k}$ such that $C \subset C_{w}$ gives a new segment joining $w$. Together with geodesic completeness of $X_{i}$, this implies that such $C$ will contain at least one path component of $X_{i} \backslash B\left(w, R^{\prime}+6 \Delta\right)$, and every path component of $X_{i} \backslash B\left(w, R^{\prime}+6 \Delta\right)$ is contained in at most one such $C$. (Geodesic completeness is used to ensure that no such $C$ will disappear when passing to $X_{i} \backslash B\left(w, R^{\prime}+6 \Delta\right)$.) Thus the number of new vertices in $V_{k}$ joining $w$ is bounded by the number of path components of $X_{i} \backslash B\left(w, R^{\prime}+6 \Delta\right)$. Call the vertex in $\Gamma$ corresponding to $w$ as $p_{w}$. Since no more new segments will join $w$ in subsequent steps, the degree of $p_{w}$ in $\Gamma$ equals one plus the number of new vertices in $V_{k}$ joining $w$. Translate $X_{i}$ by some $g$ so that $g \cdot w \in B(p, r)$. The number of path components in $X_{i} \backslash B\left(w, R^{\prime}+6 \Delta\right)$ equals that in $X_{i} \backslash B\left(g \cdot w, R^{\prime}+6 \Delta\right)$, which is at most the number of path components in $X_{i} \backslash B\left(p, r+R^{\prime}+6 \Delta\right)$, as $B\left(g \cdot w, R^{\prime}+6 \Delta\right) \subset B\left(p, r+R^{\prime}+6 \Delta\right)$. Hence we obtain a universal bound of the degree of $p_{w}$ in $\Gamma$, which means $\Gamma$ has bounded valence.

A tree of bounded valence with no terminal vertex is quasi-isometric to the trivalent tree. Such tree is called a bounded valence bushy tree. Therefore we have shown the following:

Theorem 5.9 If $X_{i}$ is a proper cocompact and geodesically complete CAT( 0$)$ space whose boundary $\partial X_{i}$ is homeomorphic to a Cantor set, then $X_{i}$ is quasi-isometric to a bounded valence bushy tree.

Now each of $X_{1}, X_{2}$ is quasi-isometric to a bushy tree, thus $X$ is quasi-isometric to the product of two bounded valence bushy trees, and so is $G$. Therefore we can apply a theorem by Ahlin [1, Theorem 1] on quasi-isometric rigidity of lattices in products of trees to show that a finite index subgroup of $G$ is a lattice in $\operatorname{Isom}\left(T_{1} \times T_{2}\right)$ where $T_{i}$ is a bounded valence bushy tree quasi-isometric to $X_{i}, i=1,2$. Notice that $\operatorname{Isom}\left(T_{1}\right) \times \operatorname{Isom}\left(T_{2}\right)$ is isomorphic to a subgroup of $\operatorname{Isom}\left(T_{1} \times T_{2}\right)$ of index 1 or 2 (which can be proved similarly as Lemma 2.1 ), we finally proved the last statement of Theorem 1.2.

\section{References}

[1] A R Ahlin, The large scale geometry of products of trees, Geom. Dedicata 92 (2002) 179-184 MR1934017

[2] W Ballmann, S Buyalo, Periodic rank one geodesics in Hadamard spaces, from: "Geometric and probabilistic structures in dynamics", Contemp. Math. 469, Amer. Math. Soc. (2008) 19-27 MR2478464 
[3] M R Bridson, A Haefliger, Metric spaces of non-positive curvature, Grundl. Math. Wissen. 319, Springer, Berlin (1999) MR1744486

[4] M Burger, S Mozes, Lattices in product of trees, Inst. Hautes Études Sci. Publ. Math. (2000) 151-194 MR1839489

[5] P-E Caprace, F Haglund, On geometric flats in the CAT(0) realization of Coxeter groups and Tits buildings, Canad. J. Math. 61 (2009) 740-761 MR2541383

[6] P-E Caprace, N Monod, Isometry groups of non-positively curved spaces: Structure theory, J. Topol. 2 (2009) 661-700 MR2574740

[7] K Fujiwara, K Nagano, T Shioya, Fixed point sets of parabolic isometries of CAT(0) spaces, Comment. Math. Helv. 81 (2006) 305-335 MR2225630

[8] M Kapovich, B Kleiner, The weak hyperbolization conjecture for 3-dimensional CAT(0)-groups, Groups Geom. Dyn. 1 (2007) 61-79 MR2294248

[9] J F Manning, Geometry of pseudocharacters, Geom. Topol. 9 (2005) 1147-1185 MR2174263

[10] P Ontaneda, Some remarks on the geodesic completeness of compact nonpositively curved spaces, Geom. Dedicata 104 (2004) 25-35 MR2043952

[11] P Papasoglu, E Swenson, Boundaries and JSJ decompositions of CAT(0)-groups, Geom. Funct. Anal. 19 (2009) 559-590 MR2545250

[12] KE Ruane, Dynamics of the action of a CAT(0) group on the boundary, Geom. Dedicata 84 (2001) 81-99 MR1825346

[13] K Ruane, CAT(0) groups with specified boundary, Algebr. Geom. Topol. 6 (2006) 633-649 MR2220692

[14] E L Swenson, A cut point theorem for CAT(0) groups, J. Differential Geom. 53 (1999) 327-358 MR1802725

Department of Mathematics, Indiana University

Bloomington, IN 47405, USA

khchao@indiana.edu

Received: 12 September 2012 Revised: 22 April 2013 